\title{
Dynamics of plantar foot temperature after conductive cold provocation in diabetic patients and healthy controls
}

\author{
by A. Seixas ${ }^{* . * *}$, J. Azevedo*, K. Ammer ${ }^{* * *}$, R. Carvalho*** , J.P. Vilas-Boas ${ }^{* \star * *}$, J. Mendes ${ }^{* * * * * *}$ \\ and R. Vardasca ${ }^{\star \star * * * \star}$
}

\author{
*Escola Superior de Saúde, Universidade Fernando Pessoa, Porto, Portugal, aderito@ufp.edu.pt \\ **LABIOMEP, INEGI-LAETA, Faculdade de Desporto, Universidade do Porto, Porto, Portugal \\ ${ }^{* * *}$ Medical Imaging Research Unit, University of South Wales, Pontypridd, United Kingdom \\ **** Hospital Santo António, Centro Hospitalar do Porto, E.P.E., Porto, Portugal \\ $\star * * \star *$ LABIOMEP, CIFI2D, Faculty of Sports, University of Porto, Porto, Portugal

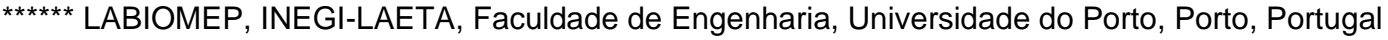

\begin{abstract}
Cold provocation tests are largely used in clinical and research settings, but conductive cold provocation tests have not been applied in the feet. This study analyses skin temperature dynamics after a conductive cold provocation test in a sample of 30 diabetic patients and 30 healthy controls. The test was easy and practical to apply and induced significant temperature changes immediately after and 5 minutes after the test. Tsk dynamics were similar in both groups. The cooling of the plantar surface was not homogenous with large differences in TSk drops between some regions of interest.
\end{abstract}

\section{Introduction}

Diabetes is a long-term condition occurring with high levels of blood glucose due to the inability to produce insulin in adequate amount or impaired ability to use the insulin that is produced in the body. High levels of glucose in the blood may lead to significant damage to most of the body's systems, but especially the nerves and blood vessels [1]. Moreover, the potential to result in blood vessels and nerves increases the risk of foot ulcers [2], which in turn increases the risk of amputation. The prevalence of diabetes is increasing worldwide and, in Europe, 1 in 11 adults have diabetes and the diabetes-related expenditure is 161 billion dollars [1]. Therefore, research on innovative approaches to detect early signs of complications are needed.

An angiosome may be defined as a three-dimensional territory of tissues that are supplied by specific arteries [3]. The plantar surface of the foot is supplied by the posterior tibial artery (PTA) and the peroneal artery (PA). The lateral border of the ankle and heel are supplied by the PA and the remaining plantar surface of the foot are supplied by three branches of the PTA, the medial calcaneal artery (MCA), the medial plantar artery (MPA) and the lateral plantar artery (LPA) [4-7].

Dynamic thermography allows to assess not only spatial characteristics of skin temperature (TSk) but also temporal behaviour of TSk in different situations. Active dynamic thermography implies the use of external stress to induce TSk changes and evaluate its effect during a time period. External stress sources can be thermal, mechanical or chemical in nature. During a thermal stress test a body part is subjected to a thermal challenge in which the region to be examined is heated or cooled and the temperature recovery is assessed.

Literature regarding the application of cold stress tests in the feet of diabetic patients is scarce and refers mainly to tests involving the immersion of the feet in water. The test requires a recipient with water at a prespecified temperature and thin plastic bags to avoid contact between water and skin, that must be removed before the assessment [8]. These requirements may become impractical and research on alternative tests is on demand. Conductive cold provocation has been used in research [9] but not in the foot. Therefore, the aim of this study was to analyse the TSk dynamics after a conductive cold provocation test in the feet of diabetic patients and healthy controls.

\section{Methodology}

The study was approved by the ethical committee of the hospital where the data collection took place. The study population was limited to diabetic patients and healthy controls older than 18 years. Participants were excluded if they decided not to cooperate, if they had major amputations, active ulcers or cognitive impairment. Participants were recruited from a specialized centre and from the local university. All participants read and signed the informed consent form before the study.

\subsection{Participants}

The sample consisted of 30 diabetic subjects with neuropathy ( 26 males) with mean age of $62.3 \pm 10.2$ years and mean body mass index (BMI) of $27.8 \pm 4.2 \mathrm{~kg} / \mathrm{m}^{2}$, and 30 healthy controls with mean age of $26.6 \pm 11.3$ years and mean body mass index (BMI) of $24.3 \pm 3.1 \mathrm{~kg} / \mathrm{m}^{2}$. The analysis was performed the foot level; therefore 120 feet were analysed. 


\subsection{Procedures}

After signing the informed consent form, patients removed the shoes and socks and remained seated with extended knees and legs supported by a chair during 10 minutes for acclimation. Room temperature $(22.4 \pm 1.3 \stackrel{\circ}{\circ})$ and relative humidity $(45.6 \pm 9.7 \%)$ were recorded during the assessments.

\subsubsection{Skin Temperature Assessment}

All TSk measurements were conducted in the morning, away from airflow and infrared radiation sources, after the 10-minute acclimation period. Images from the plantar aspect of the feet were obtained using and infrared camera (FLIR Systems, E60, Wilsonville, OR, USA) with sensor array size of $320 \times 240$, noise equivalent temperature difference (NETD) of $50 \mathrm{mK}$ at $30{ }^{\circ} \mathrm{C}$ and $\pm 2 \%$ of repeatability of the overall reading with emissivity set to 0.98 . The camera was switched on at least 40 minutes before the first data collection of the day to allow sensor stabilization. The camera was positioned perpendicular to the feet at 1 metre distance. Images were analysed with FLIR ResearchIR Max software (FLIR Systems, version 4.30.0.69) and regions of interest (ROI) were defined according to the angiosome concept (figure 1), which is a reliable methodology of infrared image analysis [10].

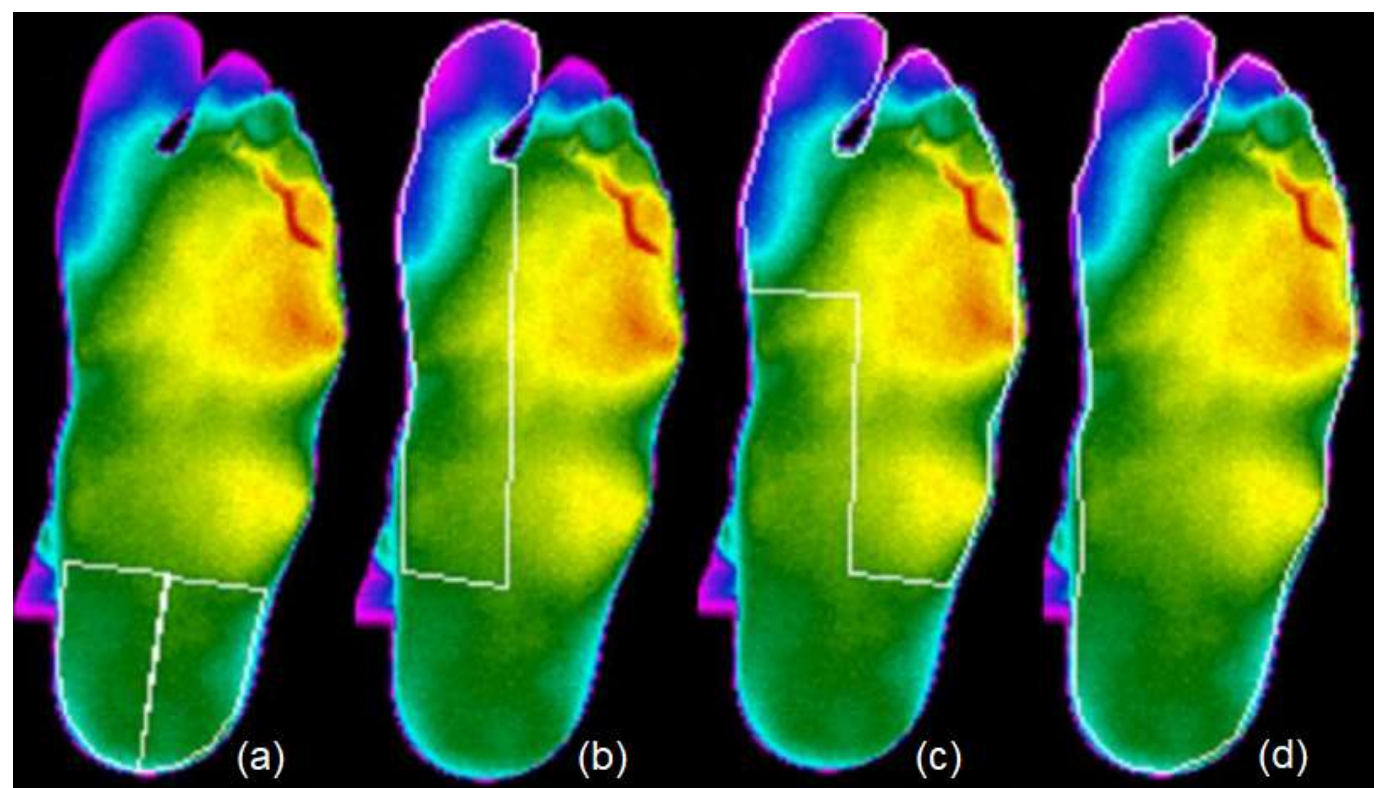

Fig. 1. Regions of interest: $M C A$ and $P A(a), M P A(b), L P A(c)$ and plantar surface of the foot (d).

Thermograms were acquired before, immediately after and 5 minutes after the conductive cold provocation test. During the test patients stood with their feet over an $8 \mathrm{~mm}$ thick aluminium plaque, that was placed on the floor, for 2 minutes (figure 2).

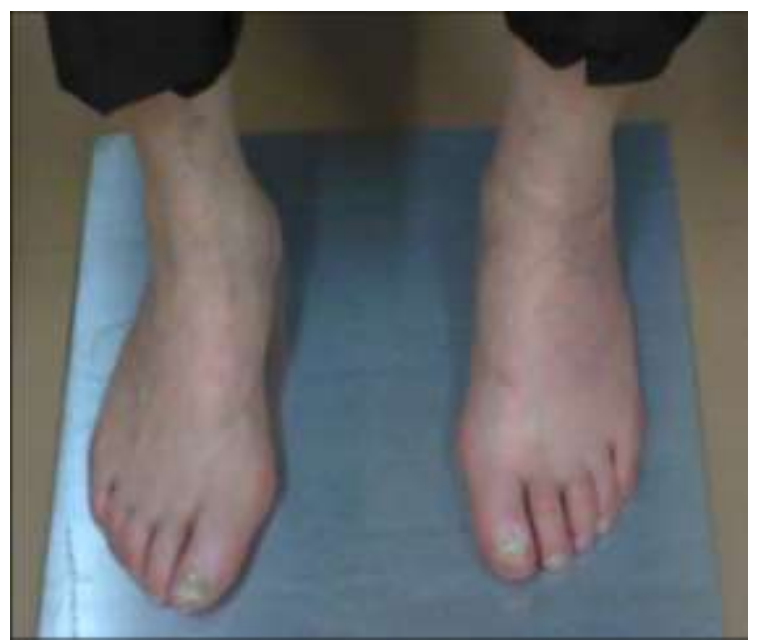

Fig. 2. Conductive cold provocation test: feet position.

\subsubsection{Data Analysis}


All data analysis was performed using Statistical Package for the Social Sciences (SPSS Statistics, IBM, version 25) and JASP (version 0.11.1). Descriptive statistics were calculated using the mean and the standard deviation. The TSk variation during the cold provocation test was analysed through repeated measures ANOVA with time (before, immediately after and 5 minutes after) as within subject factor and group (diabetics and controls) as between subject factor. TSk drop was calculated subtracting TSk before the test and TSk immediately after the test and TSk rise was calculated subtracting TSk 5 minutes fater the test and TSk immediately after the test. Independent samples T-test was used to analyse the differences in TSk drop and TSk rise in each ROI between groups. Statistical significance was considered if $p<0.05$.

\section{Results}

Sample thermograms of TSk dynamics during the cold provocation test are presented in figure 3 and the TSk dynamics in each ROI is represented in figure 4.

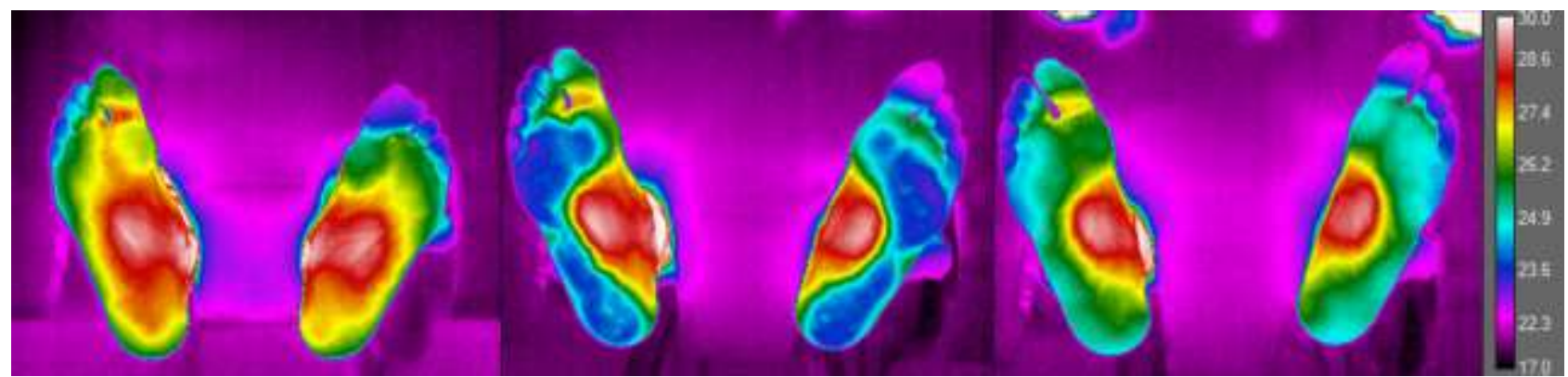

Fig. 3. TSk dynamics during the cold provocation test, before (left), after (center) and $5 \mathrm{~m}$ after (right)

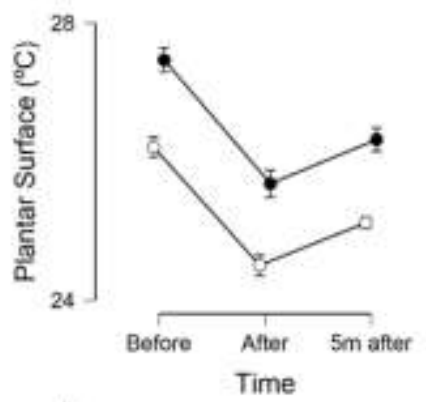

$$
\text { Group }
$$

- Diabetes

- Control
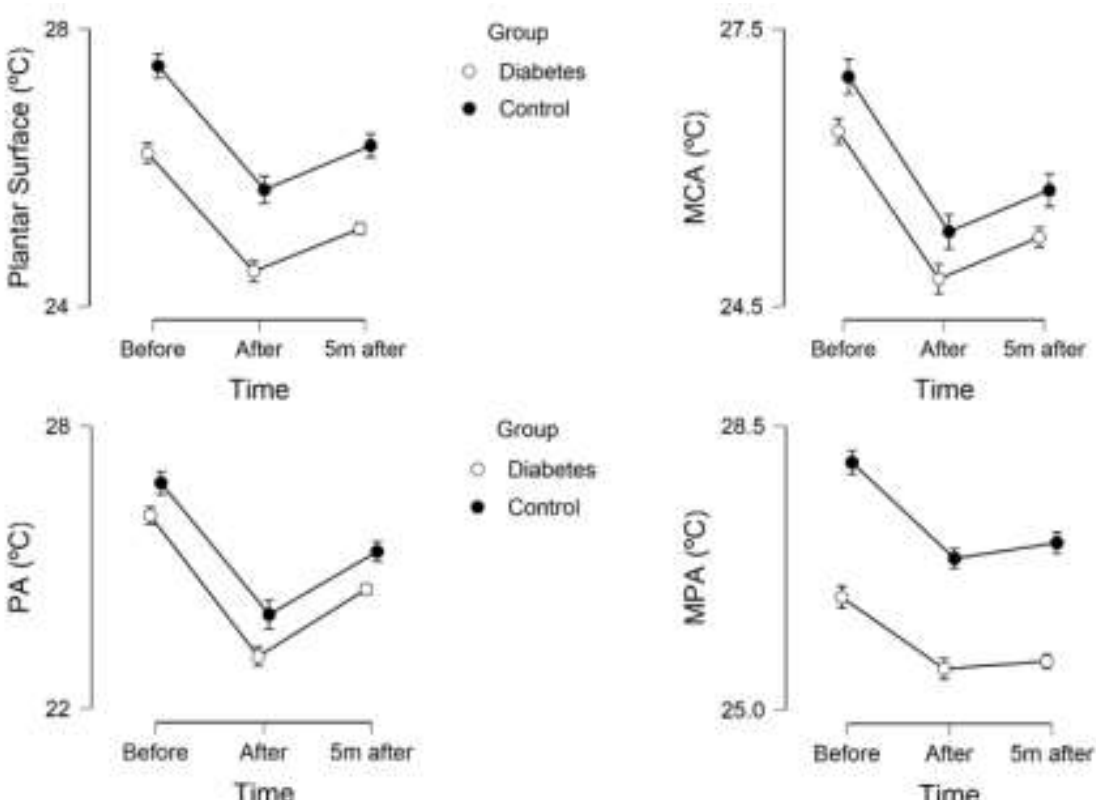

Group

Diabetes

- Control

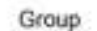

- Diabetes

- Control

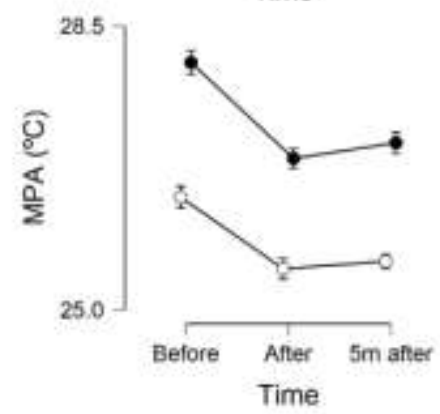

Group

- Diabetes

- Control

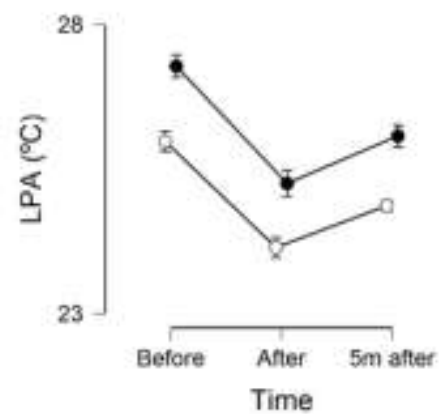

- Diabetes

- Control

Fig. 4. TSk dynamics in each ROI during the cold provocation (before, immediately after and 5 minutes after the test) 
Considering the plantar surface of the feet, there was a significant effect of Time, $F(1.87,220.42)=253.87$, $p<0.001$, but not a significant interaction between Time*Group, $F(1.87,220.42)=0.138, p=0.858$. In the MCA there was a significant effect of Time, $F(2,236)=217.44, p<0.001$, but not a significant interaction between Time ${ }^{\star} G$ roup, $F(2$, $236)=0.138, p=0.856$. In the PA there was a significant effect of Time, $F(1.63,192.86)=347.31, p<0.001$, but not a significant interaction between Time ${ }^{*}$ Group, $F(1.634,192.86)=0.409, p=0.623$. In the MPA there was a significant effect of Time, $F(1.90,224.32)=158.57, p<0.001$, but not a significant interaction between Time*Group, $F(1.90,224.32)=2.983$, $\mathrm{p}=0.055$. In the LPA there was a significant effect of Time, $F(1.82,215.01)=233.24, p<0.001$, but not a significant interaction between Time*Group, $F(1.82,215.01)=0.567, p=0.568$.

The values of TSk drop and TSk rise in each $\mathrm{ROI}$ in both groups and the results of the group comparison are presented in table 1.

Table 1. TSk dynamics: between group comparison of TSk drop and TSk rise in each ROI

\begin{tabular}{|l|l|l|l|}
\hline variable & Control & Diabetes & p-value \\
\hline TSk drop Plantar Surface & $1.8 \pm 1.1^{\circ} \mathrm{C}$ & $1.7 \pm 0.9^{\circ} \mathrm{C}$ & 0.654 \\
\hline TSk drop MCA & $1.7 \pm 1.1^{\circ} \mathrm{C}$ & $1.6 \pm 0.9^{\circ} \mathrm{C}$ & 0.660 \\
\hline TSk drop PA & $2.8 \pm 1.6^{\circ} \mathrm{C}$ & $3.0 \pm 1.2^{\circ} \mathrm{C}$ & 0.448 \\
\hline TSk drop MPA & $1.2 \pm 0.7{ }^{\circ} \mathrm{C}$ & $0.9 \pm 0.8^{\circ} \mathrm{C}$ & $0.032^{*}$ \\
\hline TSk drop LPA & $2.0 \pm 1.2^{\circ} \mathrm{C}$ & $1.8 \pm 1.1^{\circ} \mathrm{C}$ & 0.355 \\
\hline TSk rise Plantar Surface & $0.6 \pm 1.0^{\circ} \mathrm{C}$ & $0.6 \pm 0.6^{\circ} \mathrm{C}$ & 0.877 \\
\hline TSk rise MCA & $0.5 \pm 1.0^{\circ} \mathrm{C}$ & $0.5 \pm 0.9^{\circ} \mathrm{C}$ & 1.000 \\
\hline TSk rise PA & $1.3 \pm 1.5^{\circ} \mathrm{C}$ & $1.4 \pm 0.9^{\circ} \mathrm{C}$ & 0.675 \\
\hline TSk rise MPA & $0.2 \pm 0.7{ }^{\circ} \mathrm{C}$ & $0.1 \pm 0.5^{\circ} \mathrm{C}$ & 0.356 \\
\hline TSk rise LPA & $0.8 \pm 1.2^{\circ} \mathrm{C}$ & $0.7 \pm 0.7{ }^{\circ} \mathrm{C}$ & 0.581 \\
\hline
\end{tabular}

Significant differences were found only in the MPA with significantly higher TSk drop in the control group $(p=0.032)$. Graphical representations of these differences are presented in figure 5.
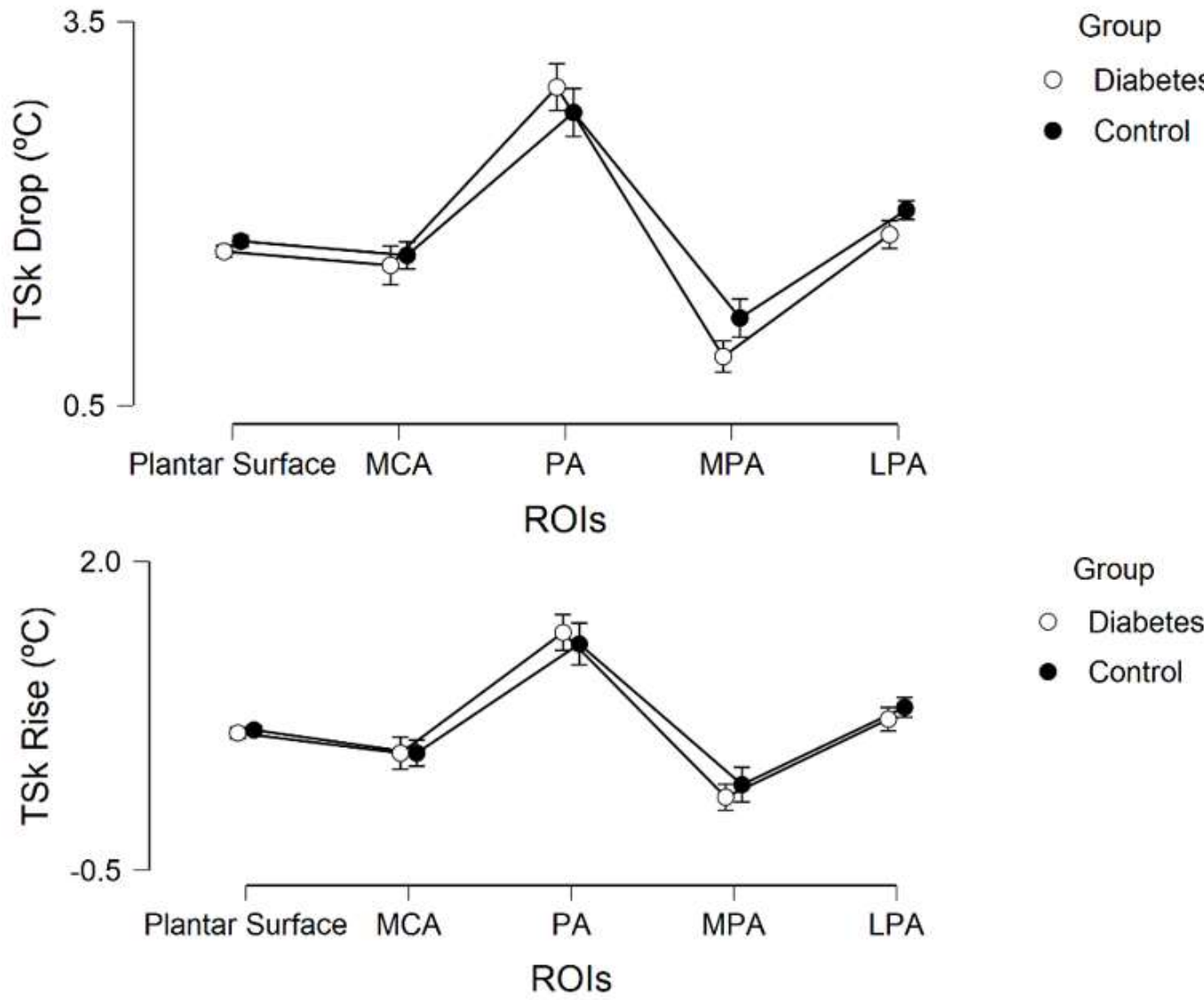

Fig. 5. TSk dynamics: between group comparison of TSk drop and TSk rise in each ROI 
TSk drop was lower in the MPA angiosome, followed by the MCA territory, and higher in the PA angiosome, followed by the LPA territory. Regarding rewarming of the foot, TSk rise was lower in the MPA angiosome, followed by the MCA territory, and higher in the PA angiosome, followed by the LPA territory. TSk dynamics were identical in both groups.

\section{Conclusion}

The proposed conductive cold provocation test was easy and practical to apply with no special requirements. Data suggests that the cooling of the plantar surface is not homogenous, with large differences between foot regions. This may be explained by the foot morphology, specifically the longitudinal plantar arch that prevents part of the medial part of the foot to be in contact with the aluminium plaque. This may be exacerbated in subjects with foot deformities, a common finding in patients with diabetes. TSk dynamics were similar in diabetic patients and healthy controls.

Further research should explore the accuracy of the test to differentiate clinical findings such as peripheral artery disease o neuropathy in patients with diabetes.

\section{REFERENCES}

[1] International Diabetes Federation, IDF Diabetes Atlas, 9th edition ed. Brussels, Belgium: International Diabetes Federation, 2019.

[2] World Health Organization, "Global report on diabetes," Geneva2016.

[3] Taylor, G. I. and Palmer, J. H., "The vascular territories (angiosomes) of the body: experimental study and clinical applications," British Journal of Plastic Surgery, vol. 40, pp. 113-141, 1987/03/01 1987.

[4] Attinger, C. E., Evans, K. K., Bulan, E., Blume, P., and Cooper, P., "Angiosomes of the foot and ankle and clinical implications for limb salvage: reconstruction, incisions, and revascularization," Plastic and reconstructive surgery, vol. 117, pp. 261S-293S, 2006.

[5] Kagaya, Y., Ohura, N., Suga, H., Eto, H., Takushima, A., and Harii, K., "'Real angiosome'assessment from peripheral tissue perfusion using tissue oxygen saturation foot-mapping in patients with critical limb ischemia," European Journal of Vascular and Endovascular Surgery, vol. 47, pp. 433-441, 2014.

[6] Nagase, T., Sanada, H., Takehara, K., Oe, M., lizaka, S., Ohashi, Y., et al., "Variations of plantar thermographic patterns in normal controls and non-ulcer diabetic patients: novel classification using angiosome concept," Journal of Plastic, reconstructive \& aesthetic Surgery, vol. 64, pp. 860-866, 2011.

[7] Alexandrescu, V.-A. and Triffaux, F., "Ischemic Ulcer Healing: Does Appropriate Flow Reconstruction Stand for All That We Need?," in Wound Healing-New insights into Ancient Challenges, ed: InTech, 2016, pp. $247-277$.

[8] Balbinot, L. F., Robinson, C. C., Achaval, M., Zaro, M. A., and Brioschi, M. L., "Repeatability of infrared plantar thermography in diabetes patients: a pilot study," Journal of Diabetes Science and Technology, vol. 7, pp. 11301137, 2013.

[9] Strzelecki, M., Strąkowska, M., Strąkowski, R., and Kaszuba, A., "Cold Provocation and Active Thermography in Medical Screening," Computational Methods in Science and Technology, vol. 23, pp. 65-71, 2017.

[10] Seixas, A., Azevedo, J., Pimenta, I., Ammer, K., Carvalho, R., Vilas-Boas, J. P., et al., "Skin temperature of the foot: Reliability of infrared image analysis based in the angiosome concept," Infrared Physics and Technology, vol. 92, pp. 402-408, 20182018. 